\title{
ENDOMORPHISM RINGS OF IDEALS IN A COMMUTATIVE REGULAR RING
}

\author{
ROGER WIEGAND ${ }^{1}$
}

Let $R$ be a commutative regular ring, and let $R_{*}$ be the Boolean ring of idempotents in $R$. It is well known that the mappings $J \mapsto \hat{J}$ $=J \cap R_{*}$ and $I \mapsto \bar{I}=R I$ are reciprocal lattice isomorphisms between ideals of $R$ and ideals of $R_{*}$. In this paper we make these mappings in to functors: To each $\phi \in \operatorname{Hom}_{R}\left(J_{1}, J_{2}\right)$, respectively $\Psi \in \operatorname{Hom}_{R_{*}}\left(I_{1}, I_{2}\right)$, we associate a homomorphism $\hat{\phi} \in \operatorname{Hom}_{R_{*}}\left(\hat{J}_{1}, \hat{J}_{\mathbf{2}}\right)$, respectively $\bar{\Psi} \in \operatorname{Hom}_{R}\left(\bar{I}_{1}, \bar{I}_{2}\right)$. Using these constructions, we prove that the endomorphism ring of an ideal of $R$ is regular, and that the homological dimension of an ideal is equal to its homological dimension as a module over its endomorphism ring.

For each $x \in R$ let $x_{*}$ be the (unique) idempotent such that $R x_{*}$ $=R x$. We will use the following relations repeatedly:

$$
x_{*} x=x, \quad(x y)_{*}=x_{*} y_{*}, \quad \forall x, y \in R .
$$

Let $\phi$ and $\Psi$ be as above, and define $\hat{\phi}: \hat{J}_{1} \rightarrow \hat{J}_{2}$ and $\bar{\Psi}: \bar{I}_{1} \rightarrow \bar{I}_{2}$ as follows:

$$
\begin{aligned}
\hat{\phi}(e) & =\phi(e)_{*}, & & \forall e \in \hat{J}_{1}, \\
\bar{\Psi}(x) & =x \Psi\left(x_{*}\right), & & \forall x \in \bar{I}_{1} .
\end{aligned}
$$

Let $\mathfrak{g}$, respectively $\mathfrak{I}_{*}$, denote the category of $R$-ideals and $R$-homomorphisms, respectively $R_{*}$-ideals and $R_{*}$-homomorphisms.

THEOREM 1. The mappings $\phi \mapsto \hat{\phi}$ and $\Psi \mapsto \bar{\Psi}$ are functors. Moreover $\bar{\Psi}^{\wedge}=\Psi$, so that $\mathfrak{G}_{*}$ is a retract of $\mathfrak{g}$.

Since we are dealing with two distinct additive structures, we use the symbol $\oplus$ to denote addition in the Boolean ring $R_{*}$. Then $e_{1} \oplus e_{2}$ $=e_{1}+e_{2}-2 e_{1} e_{2}$. Often it is convenient to consider the join of two idempotents: $e_{1} \bigvee e_{2}=e_{1} \oplus e_{2} \oplus e_{1} e_{2}=e_{1}+e_{2}-e_{1} e_{2}$. Note that $e_{1} \oplus e_{2}$ $=e_{1}\left(1-e_{2}\right) \bigvee e_{2}\left(1-e_{1}\right)$.

Lemma 1. Let $I_{1}$ and $I_{2}$ be ideals in the Boolean ring $\left(R_{*}, \oplus\right)$, and let $h: I_{1} \rightarrow I_{2}$ be any mapping. Consider the following three statements:

(a) $h\left(e_{1} e_{2}\right)=e_{1} h\left(e_{2}\right), \quad \forall e_{1} \in R_{*}, e_{2} \in I_{1}$;

(b) $h\left(e_{1} e_{2}\right)=h\left(e_{1}\right) h\left(e_{2}\right), \forall e_{1}, e_{2} \in I_{1}$;

(c) $h\left(e_{1} \bigvee e_{2}\right)=h\left(e_{1}\right) \bigvee h\left(e_{2}\right), \forall e_{1}, e_{2} \in I_{1}$.

Presented to the Society, January 26, 1969; received by the editors February 4, 1969.

1 Research was supported in part by National Science Foundation Grant GP 8751. 
Then (a) implies (b), and $h \in \operatorname{Hom}_{R_{*}}\left(I_{1}, I_{2}\right)$ if and only if both (a) and (c) hold.

Proof. Assume (a), and let $e_{1}, e_{2} \in I_{1}$. Then

$$
h\left(e_{1} e_{2}\right)=h\left(e_{1} e_{2}\right) h\left(e_{1} e_{2}\right)=e_{1} h\left(e_{2}\right) e_{2} h\left(e_{1}\right)=h\left(e_{1}\right) h\left(e_{2}\right) .
$$

Next, suppose (a) and (c) are both satisfied. Then

$$
\begin{aligned}
h\left(e_{1} \oplus e_{2}\right) & =\left(1-e_{2}\right) h\left(e_{1}\right) \vee\left(1-e_{1}\right) h\left(e_{2}\right) \\
& =\left(h\left(e_{1}\right)-e_{2} h\left(e_{1}\right)\right) \vee\left(h\left(e_{2}\right)-e_{1} h\left(e_{2}\right)\right) .
\end{aligned}
$$

But (a) and (b) together imply that $e_{2} h\left(e_{1}\right)=e_{1} h\left(e_{2}\right)=h\left(e_{1}\right) h\left(e_{2}\right)$, and it follows that $h\left(e_{1} \oplus e_{2}\right)=h\left(e_{1}\right) \oplus h\left(e_{2}\right)$. Finally, if $h$ is $R$-linear, then (a) and (b) are valid, and

$$
\begin{aligned}
h\left(e_{1} \bigvee e_{2}\right) & =h\left(e_{1}\right) \oplus h\left(e_{2}\right) \oplus h\left(e_{1} e_{2}\right)=h\left(e_{1}\right) \oplus h\left(e_{2}\right) \oplus h\left(e_{1}\right) h\left(e_{2}\right) \\
& =h\left(e_{1}\right) \bigvee h\left(e_{2}\right) .
\end{aligned}
$$

Proof of Theorem 1. It is easily seen, using (1), that $\hat{\phi}: \hat{J}_{1} \rightarrow \hat{J}_{2}$ satisfies condition (a), and hence also condition (b), of Lemma 1 . Given $e_{1}, e_{2} \in \hat{J}_{1}$, set $g=\hat{\phi}\left(e_{1}\right)+\hat{\phi}\left(e_{2}\right)-\hat{\phi}\left(e_{1} e_{2}\right)$ and $h=\phi\left(e_{1}\right)+\phi\left(e_{2}\right)$ $-\phi\left(e_{1} e_{2}\right)$. Then $h_{*}=\hat{\phi}\left(e_{1} \bigvee e_{2}\right)$ and, by condition (b), $g=\hat{\phi}\left(e_{1}\right) \bigvee \hat{\phi}\left(e_{2}\right)$. Therefore $g^{2}=g$, and a direct calculation shows that $h g=h$. If we can show that $g \in R h$ it will follow that $g=h_{*}$, and therefore that condition (c) holds. Choose $r_{1}$ and $r_{2}$ in $R$ such that $r_{i} \phi\left(e_{i}\right)=\hat{\phi}\left(e_{i}\right), i=1,2$, and let $h^{\prime}=r_{1} \hat{\phi}\left(e_{1}\right)+r_{2} \hat{\phi}\left(e_{2}\right)-r_{1} r_{2} \phi\left(e_{1} e_{2}\right)$. Then $h^{\prime} h=g$, and $g \in R h$. By Lemma $1, \hat{\phi} \in \operatorname{Hom}_{R *}\left(\hat{J}_{1}, \hat{J}_{2}\right)$. We now show that $\bar{\Psi}$ is $R$-linear. If $r \in R$ and $x \in \bar{I}_{1}$, we have $\bar{\Psi}(r x)=r x \Psi\left(r_{*} x_{*}\right)=r x \Psi\left(x_{*}\right)=r \bar{\Psi}(x)$. Now let $x, y \in \bar{I}_{1}$. Using the lemma and the fact that $\Psi$ and $\bar{\Psi}$ agree on $I_{1}$, we have

$$
\begin{aligned}
\bar{\Psi}(x+y) & =\bar{\Psi}\left((x+y)\left(x * \bigvee y_{*}\right)\right)=(x+y) \bar{\Psi}\left(x * \bigvee y_{*}\right) \\
& =(x+y)\left(\bar{\Psi}\left(x_{*}\right) \bigvee \bar{\Psi}\left(y_{*}\right)\right) \\
& =(x+y)\left(\bar{\Psi}\left(x_{*}\right)+\bar{\Psi}\left(y_{*}\right)-\bar{\Psi}\left(x_{*} y_{*}\right)\right)=\bar{\Psi}(x)+\bar{\Psi}(y) .
\end{aligned}
$$

The verification that $\hat{\phi}$ and $\bar{\Psi}$ are natural is routine and will be omitted. Clearly $\vec{\Psi}^{\wedge}=\Psi$, and the proof is complete.

The functors in Theorem 1 have the peculiar property of being "exact" but not adiditive. (Of course $\mathscr{g}$ and $\mathfrak{g}_{*}$ are not exact categories.) For example, let $R$ be a field of characteristic different from 2, and let $\phi$ and $\Psi$ be the identity maps on $R$ and $R_{*}$, respectively. Then $(\phi+\phi)^{\wedge} \neq \hat{\phi} \oplus \hat{\phi}$, and $(\Psi \oplus \Psi)^{-} \neq \bar{\Psi}+\bar{\Psi}$. This apparent unnaturality is explained by Theorem 2 below. 
Lemma 2. Let $J$ and $K$ be ideals of the commutative regular ring $R$, and assume that $K \subseteq J$. Then $\phi K \subseteq K$ for each $\phi \in \operatorname{Hom}_{R}(J, J)$. In particular, if $S=\operatorname{Hom}_{R}(J, J)$ then $K$ is a left $S$-module.

Proof. If $x \in K, \phi(x)=x \phi\left(x_{*}\right) \in K$.

The following lemma describes explicitly the "image" of the functor $\Psi \mapsto \bar{\Psi}$ :

Lemma 3. Let $\phi \in \operatorname{Hom}_{R}(J, J)$. The following conditions are equivalent: (a) $\phi=\hat{\phi}^{-}$; (b) $\phi=\bar{\Psi}$ for some $\Psi \in \operatorname{Hom}_{R_{*}}(\hat{J}, \hat{J})$; (c) $\phi^{2}=\phi$; (d) $\phi \hat{J} \subseteq \hat{J}$.

Proof. First observe that the composition in the ring $S$ $=\operatorname{Hom}_{R}(J, J)$ may be described by

$$
\left(\phi_{2} \phi_{1}\right)(x)=x \phi_{2}(x *) \phi_{1}(x *) .
$$

Now (a) obviously implies (b). To show (b) implies (c), we have

$$
\bar{\Psi}^{2}(x)=x \bar{\Psi}\left(x_{*}\right)^{2}=x \Psi(x *)=\bar{\Psi}(x) .
$$

Next, assuming (c), we have

$$
\phi(e)^{2}=e \phi(e)^{2}=\phi^{2}(e)=\phi(e),
$$

so (d) is satisfied. Finally, if (d) holds

$$
\hat{\phi}^{-}(x)=x \hat{\phi}\left(x_{*}\right)=x \phi\left(x_{*}\right) * x \phi\left(x_{*}\right)=\phi(x) ;
$$

therefore (d) implies (a), and the proof is complete.

THEOREM 2. Let $J$ be an ideal in the commutative regular ring $R$, let $S=\operatorname{Hom}_{R}(J, J)$, and let $\hat{S}=\operatorname{Hom}_{R_{*}}(\hat{J}, \hat{J})$. Then $\hat{S}$ is a Boolean ring, $S$ is a commutative regular ring, and the map $\theta: \Psi \mapsto \bar{\Psi}$ is a ring isomorphism from $\hat{S}$ onto $S_{*}$, the Boolean ring of idempotents in $S$.

Proof. By (3), $S$ is commutative. Given $\phi \in S$, let $\phi_{*}=\hat{\phi}^{-}$. By Lemma $3, \phi_{*}$ is idempotent, and a simple computation using (3) shows that $\phi_{*} \phi=\phi$. We will show that $S \phi_{*}=S \phi$.

For each $x \in R$ there is a unique $\tilde{x} \in R$ satisfying the relations

$$
x \tilde{x}=x_{*}, \quad x_{*} \tilde{x}=\tilde{x} .
$$

(Choose $y$ such that $x y x=x$ and set $\tilde{x}=y x y$.) Define $\tilde{\phi}: J \rightarrow J$ by letting $\tilde{\phi}(x)=x \phi\left(x_{*}\right)^{\sim}$. Now clearly

$$
(x y)^{-}=\tilde{x} \tilde{y}, \quad \forall x, y \in R ; \quad \tilde{e}=e, \quad \forall e \in R *
$$

It follows from (1) and (5) that

$$
\tilde{\phi}(r x)=r \tilde{\phi}(x), \quad \forall r \in R, \quad x \in J
$$


and

$$
\tilde{\phi}(e)=\phi(e)^{\sim}, \quad \forall e \in \hat{J} .
$$

Let $e_{1}, e_{2} \in \hat{J}$ and set $s=\tilde{\phi}\left(e_{1}\right)+\tilde{\phi}\left(e_{2}\right)-\tilde{\phi}\left(e_{1} e_{2}\right)$. By (6) and (7) we may write $s=e_{1} \phi\left(e_{1}\right)^{\sim}+e_{2} \phi\left(e_{2}\right)^{\sim}-e_{1} e_{2} \phi\left(e_{1} e_{2}\right)^{\sim}$. Since $e_{i}\left(e_{1} \bigvee e_{2}\right)=e_{i}, i=1,2$, and $e_{1} e_{2}\left(e_{1} \bigvee e_{2}\right)=e_{1} e_{2}$, we have

$$
\begin{aligned}
s \phi\left(e_{1} \bigvee e_{2}\right) & =\phi\left(e_{1} \tilde{\phi} \phi\left(e_{1}\right)+\phi\left(e_{2}\right) \tilde{\phi}\left(e_{2}\right)-\phi\left(e_{1} e_{2} \tilde{)} \phi\left(e_{1} e_{2}\right)\right.\right. \\
& =\hat{\phi}\left(e_{1}\right)+\hat{\phi}\left(e_{2}\right)-\hat{\phi}\left(e_{1} e_{2}\right)=\hat{\phi}\left(e_{1}\right) \bigvee \hat{\phi}\left(e_{2}\right) \\
& =\hat{\phi}\left(e_{1} \bigvee e_{2}\right)=\phi\left(e_{1} \bigvee e_{2}\right) * .
\end{aligned}
$$

A similar calculation shows that $s \phi\left(e_{1} \bigvee e_{2}\right)_{*}=s$. We conclude that $s=\phi\left(e_{1} \bigvee e_{2}\right)^{\sim}$. Taking (7) into account, we have

$$
\tilde{\phi}\left(e_{1} \vee e_{2}\right)=\tilde{\phi}\left(e_{1}\right)+\tilde{\phi}\left(e_{2}\right)-\tilde{\phi}\left(e_{1} e_{2}\right) .
$$

Now if $x, y \in J$,

$$
\tilde{\phi}(x+y)=(x+y) \tilde{\phi}\left(x * \bigvee y_{*}\right)=\tilde{\phi}(x)+\tilde{\phi}(y), \quad \text { by (6) and (8). }
$$

Therefore $\phi \in \operatorname{Hom}_{R}(J, J)$. One checks directly that $\phi \tilde{\phi}=\phi_{*}$ and $\phi_{*} \tilde{\phi}=\tilde{\phi}$. Hence $S \phi_{*}=S \phi$, and $S$ is regular.

It remains to be shown that $\theta$ is a ring isomorphism. Clearly $\bar{\Psi}=0$ implies $\Psi=0$, and $\theta$ is onto by Lemma 3. If $\Psi_{1}$ and $\Psi_{2}$ are in $\hat{S}$ then $\left(\Psi_{2} \Psi_{1}\right)^{-}=\bar{\Psi}_{2} \bar{\Psi}_{1}$ by Theorem 1 . Therefore we need only verify that $\left(\Psi_{1} \oplus \Psi_{2}\right)^{-}=\bar{\Psi}_{1} \oplus \bar{\Psi}_{2}$, (where the symbol $\oplus$ denotes addition in both $\hat{S}$ and $\left.S_{*}\right)$. Let $x \in J$. Then

$$
\begin{aligned}
\left(\Psi_{1} \oplus \Psi_{2}\right)^{-}(x) & =x\left(\Psi_{1} \oplus \Psi_{2}\right)\left(x_{*}\right)=x\left(\Psi_{1}\left(x_{*}\right) \oplus \Psi_{2}(x *)\right) \\
& =x\left(\Psi_{1}\left(x_{*}\right)+\Psi_{2}\left(x_{*}\right)-2 \Psi_{1}\left(x_{*}\right) \Psi_{2}\left(x_{*}\right)\right) \\
& =x \Psi_{1}\left(x_{*}\right)+x \Psi_{2}\left(x_{*}\right)-2 x \bar{\Psi}_{1}\left(x_{*}\right) \bar{\Psi}_{2}\left(x_{*}\right) \\
& =\bar{\Psi}_{1}(x)+\bar{\Psi}_{2}(x)-2 \bar{\Psi}_{1} \bar{\Psi}_{2}(x) \\
& =\left(\bar{\Psi}_{1}+\bar{\Psi}_{2}-2 \bar{\Psi}_{1} \bar{\Psi}_{2}\right)(x)=\left(\bar{\Psi}_{1} \oplus \bar{\Psi}_{2}\right)(x) \text {. Q.E.D. }
\end{aligned}
$$

Remark. One can also use Pierce's sheaf-theoretic representation of commutative regular rings [4] to prove that $S$ is regular. It can be shown [6] that $S$ is isomorphic to $\Gamma(U[J], R(R))$. If

$$
\sigma \in \Gamma(U[J], R(R)),
$$

define $\tilde{\sigma}: U[J] \rightarrow R(R)$ by letting $\tilde{\sigma}(x)=\sigma(x)^{-1}$ if $\sigma(x) \neq 0$, and $\sigma(x)=0$ otherwise. Then $\tilde{\sigma}$ is continuous (this requires proof) and $\sigma \tilde{\sigma} \sigma=\sigma$, that is, $\Gamma(U[J], \Re(R))$ is regular. Theorem 1 has a similar proof.

We now return to the situation described in Lemma 2 and consider 
the homological dimension of $K$ as an $S$-module. Notice that $K$ may be identified with an ideal of $S$.

Theorem 3. Let $R, J, K$ and $S$ be as in Lemma 2. Then $h \cdot \operatorname{dim}_{R}(K)$ $=$ h.dim ${ }_{S}(K)$.

Proof. We use a modification of Pierce's projective resolution for ideals in a Boolean ring [5]. Let $\left\{e_{i}: i \in I\right\}$ be a set of idempotent generators for $K$. If $\delta=\left(i_{0} \cdots i_{n}\right) \in I^{n+1}$, let $e_{\delta}$ denote the product $e_{i_{0}} \cdots e_{i_{n}}$. For each $n \geqq 0$ let $P_{n}$ be the $R$-module of all functions $\phi: I^{n+1} \rightarrow R$ such that (i) $\phi(\delta)=0$ for all but finitely many $\delta \in I^{n+1}$ and (ii) $\phi(\delta) \in \operatorname{Re}_{\delta}$ for each $\delta \in I^{n+1}$. If $\delta \in I^{n+1}$, let $x_{\delta} \in P_{n}$ be defined by

$$
\begin{array}{ll}
x_{\delta}\left(\delta^{\prime}\right)=0, & \delta^{\prime} \neq \delta, \\
x_{\delta}\left(\delta^{\prime}\right)=e_{\delta}, & \delta^{\prime}=\delta .
\end{array}
$$

Then $P_{n}=\oplus \sum_{\delta \in I^{n+1}} R x_{\delta}$, and, since $R x_{\delta} \cong R e_{\delta}, P_{n}$ is projective. Define $\epsilon: P_{0} \rightarrow K$ by letting $\epsilon(\phi)=\sum_{i \in I} \phi(i)$. For each $\delta=\left(i_{0} \cdots i_{n}\right) \in I^{n+1}$, let $\delta_{j}=\left(i_{0} \cdots \hat{\imath}_{j} \cdots i_{n}\right)$, and define $\partial x_{\delta}=e_{\delta} \sum_{0 \leq j \leq n}(-1)^{j} x_{\delta j}$. Clearly $\partial$ may be extended to a unique $R$-homomorphism $\partial: P_{n} \rightarrow P_{n-1}$. The sequence

$$
P_{*}: \cdots \rightarrow P_{n} \stackrel{\partial}{\rightarrow} P_{n-1} \rightarrow \cdots \rightarrow P_{1} \stackrel{\partial}{\rightarrow} P_{0} \stackrel{\epsilon}{\rightarrow} K \rightarrow 0
$$

is the desired projective resolution of $K$. The proof of exactness is very similar to the proof given in [5] and will be omitted.

Given $h \in S, \phi \in P_{n}$ and $\delta \in I^{n+1}$, let $(h \phi)(\delta)=h(\phi(\delta))$. By Lemma 2, $h \phi \in P_{n}$, so that $P_{n}$ is an $S$-module. Now $R x_{\delta}$ is an $S$-submodule of $P_{n}$; in fact $R x_{\delta} \cong S h_{\delta}$, where $h_{\delta} \in S$ is defined by $h_{\delta}(x)=e_{\delta} x$. Therefore $P_{n}$ is a projective $S$-module. The proof that $\partial$ and $\epsilon$ are $S$-homomorphisms presents no difficulty since it is enough to check linearity on each direct summand. Therefore $P_{*}$ is an $S$-projective resolution of $K$. For each $n \geqq 0$ let $A_{n}=\operatorname{im}\left(\partial: P_{n+1} \rightarrow P_{n}\right)$. In view of [1, VI., 2.1] the proof will be complete once we verify that $A_{n}$ is $R$-projective if and only if it is $S$-projective.

Suppose $A_{n}$ is $R$-projective. Since $A_{n}$ is generated by $\left\{\partial x_{\delta}: \delta \in I^{n+2}\right\}$ there are idempotents $g_{\delta} \in R$ such that $A_{n}=\oplus \sum_{\delta \in I^{n+2}} R g_{\delta} \partial x_{\delta}$, by [5, 3.1]. But $R g_{\delta} \partial x_{\delta}=g_{\delta} \partial R x_{\delta}=g_{\delta} \partial S x_{\delta}=S g_{\delta} \partial x_{\delta}$, so that

$$
A_{n}=\oplus \sum_{\delta \in I^{n+2}} S g_{\delta} \partial x_{\delta} .
$$

Now $S g_{\delta} \partial x_{\delta}$ is a cyclic submodule of the projective $S$-module $P_{n}$. Since $S$ is semihereditary $S g_{\delta} \partial x_{\delta}$ must be $S$-projective $[1, \mathrm{I} ., 6.2]$; therefore 
$A_{n}$ is $S$-projective. Conversely, if $A_{n}$ is $S$-projective there are idempotents $f_{\delta} \in S$ such that $A_{n}=\oplus \sum_{\delta \in I^{n+2}} S f_{\delta} \partial x_{\delta}$. But $S f_{\delta} \partial x_{\delta}=f_{\delta} \partial S x_{\delta}$ $=f_{\delta} \partial R x_{\delta}=R f_{\delta} \partial x_{\delta}$. As before, it follows that $P_{n}$ is $R$-projective. Q.E.D.

If $J$ is any ideal of $R$ and $S=\operatorname{Hom}_{R}(J, J)$ let $\mu: R \rightarrow S$ be the ring homomorphism $x \rightarrow \mu_{x}$, where $\mu_{x}(y)=x y$ for each $y \in J$. Any $S$-module acquires an $R$-module structure via this homomorphism. In view of Theorem 3 one might be led to conjecture that the homological dimension of any $S$-module is equal to its homological dimension as an $R$-module. We shall show that in fact either dimension may dominate the other.

EXAmple 1. Let $J=\oplus \sum_{n<\omega} R_{n}$, where each $R_{n}$ is a two-element Boolean ring. Embed $J$ as a maximal ideal in the Boolean ring $R$ [2, Exercise 1.1]. Then $S=\operatorname{Hom}_{R}(J, J) \cong \prod_{n<\omega} R_{n}$. Now $R$ is countable and therefore hereditary. I claim that $S$ is not hereditary. Since $S$ is complete as a Boolean algebra it must be a self-injective ring, by $[4,24.2]$ and $[2, \S 21]$. But the only self-injective hereditary rings are semisimple with minimum condition, by [3] and [1, I, 5.4]; hence $S$ cannot be hereditary. If $A$ is any $S$-module with homological dimension at least 2, we have h.dim $S(A)>$ h.dim $R(A)$.

For an example of the opposite inequality we shall show that in general $S$ need not be $R$-projective.

Lemma 4. Let $J$ be an ideal in the commutative regular ring $R$ and let $e \in R_{*}$. Then $R e$ is an essential extension of $J$ if and only if $(0: J)$ $=R(1-e)$.

The proof is straightforward and will be omitted.

Definition. An ideal $J$ of $R$ is essentially principal if $J$ is large in some principal ideal. $J$ is taut if the homomorphism $\mu: R \rightarrow \operatorname{Hom}_{R}(J, J)$ is an epimorphism.

THEOREM 4. Let $J$ be an ideal in the commutative regular ring $R$, and let $S=\operatorname{Hom}_{R}(J, J)$. Then $S$ is projective if and only if $J$ is taut and essentially principal.

Proof. Consider the sequence

$$
0 \rightarrow(0: J) \rightarrow R \stackrel{\mu}{\rightarrow} S \rightarrow 0
$$

which is clearly exact, except possibly at $S$. Suppose $S$ is projective. By $[5,3.2], \mu R$ is a direct summand of $S$. But $\mu R$ is large in $S$; therefore $\mu R=S$, so that $J$ is taut. Since $S$ is projective, (E) is split exact. 
Therefore $(0: J)$ is principal, and, by Lemma $4, J$ is essentially principal. Conversely, if $J$ is taut and essentially principal, $(0: J)$ is a direct summand of $R$, and (E) is split exact. Therefore $S$ is projective.

COROLlaRY. Let $R$ be a commutative regular ring. The following conditions are equivalent: (a) $\operatorname{Hom}_{R}(J, J)$ is projective for each ideal $J$; (b) every ideal of $R$ is taut; (c) $R$ is self-injective.

Proof. (a) implies (b) by Theorem 4. Also (b) and (c) are equivalent, by Lemma 2 and [1, I, 3.2]. Suppose (c) holds. Then the maximal ideal space of $R$ is extremally disconnected [4,24.1]; hence $R_{*}$ is complete as a Boolean algebra $[2, \S 21]$. Letting $e=\mathrm{V} \hat{\boldsymbol{J}}$, we see that $R e$ is an essential extension of $J$. By Theorem $4, \operatorname{Hom}_{R}(J, J)$ is projective. The proof is now complete.

The ideal $J$ in Example 1 is essentially principal but not taut. The following is an example of an ideal which is taut but not essentially principal.

EXAMPLE 2. Let $X_{1}$ and $X_{2}$ be disjoint copies of the space of ordinals $\leqq \Omega$ with the order topology. Let $X$ be the space obtained from $X_{1}$ $\cup X_{2}$ by identifying the two copies of the point $\Omega$. Let $U=X_{1}-\{\Omega\}$. Let $R$ be the Boolean ring of $X$ and let $J$ be the ideal corresponding to the open set $U \subseteq X$. Then $J$ is taut, since any 2 -valued function on $U$ is eventually constant. But $J$ is not essentially principal, since $U^{-}$ is not open in $X$.

Much of the work of this paper was motivated by the following question: If two commutative regular rings have homeomorphic maximal ideal spaces, do they necessarily have the same global dimension? The obvious approach is to try to prove that h.dim $\operatorname{dim}_{R}(J)$ $=$ h.dim $R_{*}(\hat{J})$, whenever $J$ is an ideal of the commutative regular ring $R$. Theorems 2 and 3 reduce the problem to the special case in which $J$ is large and taut in $R$. To see this, note that $\mu J$ is always large and taut in $S=\operatorname{Hom}_{R}(J, J)$. Our assertion now follows from

THEOREM 5. Let $J$ be an ideal in the commutative regular ring $R$, let $S=\operatorname{Hom}_{R}(J, J)$, and let $\mu: R \rightarrow$ Sbe the multiplication map.If $\operatorname{dim}_{S}(\mu J)$ $=\operatorname{h} \operatorname{dim}_{S_{*}}\left((\mu J)^{\wedge}\right)$, then h.dim $R(J)=h \cdot \operatorname{dim}_{R_{*}}(\hat{J})$.

Proof. The map $\mu$ defines an $S$-isomorphism from $J$ on to $\mu J$. Similarly, if $\hat{S}=\operatorname{Hom}_{R_{*}}(\hat{J}, \hat{J})$, we have an $\hat{S}$-isomorphism $\nu$ from $\hat{J}$ onto $\nu \hat{J} \subseteq \hat{S}$. Now it is easily checked that the ring isomorphism of Theorem 2 takes $\nu \hat{J}$ onto $(\mu J)^{\wedge}$. The desired result now follows by applying Theorem 3 to the rings $R$ and $R_{*}$. 


\section{REFERENCES}

1. H. Cartan and S. Eilenberg, Homological algebra, Princeton, N. J., 1956.

2. P. R. Halmos, Boolean algebras, Van Nostrand, Princeton, N. J., 1963.

3. B. Osofsky, Rings all of whose finitely generated modules are injective, Pacific J. Math. 14 (1964), 645-650.

4. R. S. Pierce, Modules over commutative regular rings, Mem. Amer. Math. Soc. No. 70 (1967).

5. - The global dimension of Boolean rings, J. Algebra 7 (1967), 91-99.

6. R. Wiegand, Sheaf cohomology of locally compact totally disconnected spaces, Proc. Amer. Math. Soc. 20 (1969), 533-538.

UNIVERSITY OF WISCONSIN 